\title{
Biases of Shear Wave vs Transient Elastography in fibrosis assessment of patients with fatty liver disease
}

\author{
VASILE-ANDREI OLTEANU ${ }^{1}$, GHEORGHE G. BĂLAN ${ }^{1}$, GEORGIANA EMMANUELA \\ GÎLCĂ BLĂNARIU ${ }^{1}$, OANA TIMOFTE ${ }^{1}$, DANA ELENA MITRICĂ ${ }^{1}$, SMARANDA \\ DIACONESCU ${ }^{2}$, GHEORGHE BĂLAN ${ }^{1}$, GABRIELA ȘTEFĂNESCU ${ }^{1 *}$
}

${ }^{1}$ Gastroenterology Department "Gr. T. Popa" University of Medicine and Pharmacy, Institute of Gastroenterology and Hepatology, St. Spiridon Emergency County Hospital, Iași, Romania

${ }^{2}$ Pediatrics Department, "Gr. T. Popa" University of Medicine and Pharmacy, Department of Pediatric Gastroenterology - "Sf. Maria” Clinical Emergency Children’s Hospital, Iași, Romania

\begin{abstract}
Background: The assessment of fibrosis in chronic liver diseases using non-invasive methods is an important topic in hepatology.

Aim: To compare Shear Wave and Transient elastography and in what the possible measurement biases and discordance factors are concerned.

Material and Methods: This study enrolled 139 patients with non-alcoholic fatty liver disease, who underwent assessment of hepatic fibrosis by Shear Wave and Transient elastography.

Results: The results obtained by the two methods were correlated in the most cases, but discordance in one or more degrees of fibrosis stage was also found. The multiple correlations analysis regarding the predictors of discordance indicated that a significant influence was exercised by the fibrosis degree, steatosis, BMI and necroinflammatory activity.

Conclusions: Shear Wave and Transient elastography appear as comparably reliable methods for assessment of liver fibrosis, but there are possible discrepancies in evaluating fibrosis stage.
\end{abstract}

Keywords Non-alcoholic fatty liver disease, liver fibrosis, Transient elastography, Shear Wave elastography.

Abbreviations

SWE - Shear Wave elastography; TE - Transient elastography; NASH - Non-alcoholic fatty liver disease; ROI - region-of-interest; BMI - body mass index.

To cite this article: OLTEANU V-A, BĂLAN G.GH, GÎLCĂ-BLĂNARIU G-E, TIMOFTE O, MITRICĂ D-E, DIACONESCU S, BĂLAN GH, STEFĂNESCU G. Biases of Shear Wave vs Transient Elastography in fibrosis assessment of patients with fatty liver disease. Rom Biotechnol Lett. 2020; 25(1): 1289-1295. DOI: 10.25083/rb1/25.1/1289.1295

$\square$ *Corresponding author: GABRIELA ȘTEFĂNESCU, Associate Professor - Gastroenterology Department "Gr. T. Popa" University of Medicine and Pharmacy, Institute of Gastroenterology and Hepatology, St. Spiridon Emergency County Hospital, Iași, Romania E-mail: gabriela.stefanescu@gmail.com, Tel: +40744244266, Fax: +40232222453 Home address: Clopotari 14, bl 608 B, apt 5, Iași, Romania, zip code 700723 


\section{Introduction}

Non-alcoholic fatty liver disease (NAFLD) is considered the hepatic manifestation of a metabolic syndrome and is the first cause of chronic hepatic disease being recognized as a leading cause of liver-related morbidity and mortality in many developed countries worldwide (MARCHESINI \& al [1]; ANSTEE \& al [2]; MCPHERSON \& al [3]; BELLENTANI \& al [4]; MACHADO \& al [5]; ARMSTRONG \& al [6]).

Liver fibrosis is part of the structural and functional alterations in most chronic liver diseases. The presence and extent of fibrosis is one of the main prognostic factors regarding the evaluation of progression to cirrhosis and related complications, independent on the etiology of liver disease (EKSTEDT \& al [7]; ANGULO \& al [8]).

Consequently, using a noninvasive, affordable, reproducible and high sensitivity method in order to assess liver fibrosis in NAFLD patients is currently an important topic in hepatology (NIH CONSENSUS, [9]; CASTERA \& al [10]).

Such non-invasive methods rely on two different approaches: a "biological" one based on the quantification of biomarkers in serum samples (FibroTest, FibroMeter, FibroSpectII, HepaScore, aspartate-to-platelet ratio index APRI) and a physically - based one, relying on measuring liver stiffness, which are: transient elastography, an ultrasound-based technique (acoustic radiation force impulse imaging - ARFI, point shear wave elastography pSWE, 2D-shear wave elastography - 2D-SWE) and 3-D magnetic resonance elastography. Despite the complementarity of these approaches, they rely on different rationales (RATZIU \& al [11]; EASL-ALEH CLINICAL PRACTICE GUIDELINES, [12]).

Liver stiffness assessment methods based on serum biomarkers are well validated, have a good applicability, but have the disadvantage of being expensive and of using parameters that are not specific for liver disease and, therefore, may vary more in other pathological conditions (RATZIU \& al [11]).

On the other hand, methods based on the evaluation of liver stiffness are easy to apply, they evaluate a physical parameter specific to the liver, the analytic method is simple and the cost is low (only if the device is available). Among the most validated non-invasive methods used to assess staging of liver fibrosis is transient elastography (TE), which is based on liver stiffness measurement and can be considered the non-invasive standard for the measurement of liver stiffness. There are several advantages of $\mathrm{TE}$, including short procedure time (less than 5 minutes), immediate results, and the possibility to perform it either at patient's bedside or in an outpatient clinic. This method has been well validated for viral hepatitis (B, C and in HIVVHC coinfections) but is less well validated in other chronic liver diseases (SANDRIN, [13]; FRIEDRICHRUST, [14]; MYERS, [15]; TAMANO \& al [16]).

The method has the disadvantage that it has a lower applicability than serum biomarker (obesity, ascites, operator experience), region-of-interest (ROI) cannot be chosen and requires a dedicated device damage (CASTERA \& al [17]; SIGRIST \& al [18]).

SWE is a new and promising technique based on liver assessment which is studied. Current results show that this technique is at least equivalent to TE for non-invasive staging of liver fibrosis. One advantage of SWE is its implementation on an ultrasound device and also the advantage that ROI can be adjusted in size and location and chosen by the operator (SIGRIST \& al [18]; FERRAIOLI \& al [19]; POYNARD \& al [20]).

Considering the current NAFLD guidelines, there is the need to consider performing a liver biopsy in patients at high risk for advanced fibrosis or for $\mathrm{NASH}$, and when there is not possible to exclude other etiologies for fatty liver or coexisting chronic liver disease, through non-invasive methods. However, many authors consider that first using a non-invasive method to rule out advanced fibrosis is preferable (CHALASANI \& al [21]). The present study compares two non-invasive methods of fibrosis assessment (TE and SWE) in NAFLD. We examined the predictors of discordance between TE and SWE, defined as a difference of one or more stages between techniques. The aim was to compare the applicability (the measurement of reliable results) and performances of SWE for the diagnosis of fibrosis as compared with TE applying a methodology without gold standard (liver biopsy). The study protocol is not addressed to assess the sensibility/specificity of these methods.

\section{Methods}

\section{Patients}

We performed a prospective study, including 139 patients with NAFLD, evaluated in the Institute of Gastroenterology and Hepatology - Emergency Hospital "St. Spiridon" Iasi, between January 2017 and December 2017. According to the American Association for the Study of Liver Disease guidelines, NAFLD is confirmed when the following 4 criteria are simultaneously present: (1) fatty change of the liver; (2) no other factors causing chronic liver disease are present; (3) no other factors inducing fatty change of the liver are present; (4) no significant alcohol abuse is present (CHALASANI \& al [21]).

All patients diagnosed with fattening of the liver detected by ultrasound examination (US) were included in the study. The exclusion criteria were the evidence of decompensated liver cirrhosis (Child-Pugh class B or C), other pathological conditions which may interfere with measurements results (biliary obstruction, primary biliary cirrhosis, autoimmune or viral hepatitis, metabolic or hereditary liver diseases, proteincalorie malnutrition, total parenteral nutrition, rapid weight loss, a history of intestinal resection, inflammatory bowel disease, thyroid disease, malignancy, drug use, chronic heart disease, or with vascular malformation or focal lesion detected on US, excessive alcohol consumption for more than 5 years, liver stiffness measurement failure, or unreliable Fibroscan (less than 10 valid measurments). The study was approved by the local Ethics Committee. Patients' written informed consent was obtained before enrollment.

\section{Clinical and laboratory data}

Demographic details and BMI were collected. A complete blood count and blood chemistry tests were assessed. ALAT and ASAT values for liver enzymes and total cholesterol and triglycerides (TG) values for blood lipids were measured. Prothrombin activity, fibrinogen, alkaline phosphatase, gamma glutamyl transferase were also investigated at inclusion in study. 


\section{Ultrasound exam $(U)$}

Ultrasonography examinations were performed by a single examinator. Patients were placed in supine position and examined using either subcostal or intercostal approach. The craniocaudal sizes of the left and right liver lobe were measured, on the midclavicular line, in the saggital plane. Bipolar spleen dimeter (BSD) and portal vein diameter were examined using ultrasonography. The degree of liver fattening was categorized as grade I steatosis when there was a slight increase in the diffusion of liver echogenicity and normal echogenicity of the diaphragm and intrahepatic vein walls. Patients with increased liver echogenicity, with minimal washout of intrahepatic vein walls and diaphragm echogenicity were classified as grade II steatosis. Patients with a definite increase in liver echogenicity with posterior segments of the liver not clearly visible and intrahepatic vein walls and diaphragm partly or fully obscured were evaluated as grade III steatosis.

\section{Liver Stiffness Measurement by Shear Wave elasto- graphy (SWE)}

A GE Healthcare LOGIQ E9 system with the R5 software version and the C1-6-D probe was used in order to acquire data on liver shear wave elastography. All measurements were performed by the same investigator, using the same device, during ultrasound examination.

The patients were placed in the left posterior oblique position, with the right arm over their head and the right liver lobe was assessed using intercostal approach, using the $7^{\text {th }}$ or $8^{\text {th }}$ intercostal spaces in the right anterior axillary line to perform measurements. The acquisition region-ofinterest (ROI) was selected at least at $1 \mathrm{~cm}$ below the liver capsule, with no significant vessels (if possible). During end-expiration breath holds, 10 repeated SWE measurements were performed, with each SWE acquisition requiring about 1 to 2 seconds. Following acquisitions, the measurements were performed by placing 10 circular measurement regions over the saved SWE images, each circular region accounting for approximately $1 \mathrm{~cm}$ in diameter.

The median value was considered representative and the results were expressed in kilopascals $(\mathrm{kPa})$. A SWE measurement was considered valid if the interquartile range was $<30 \%$ of the median value. An unsuccessful 2d-SWE acquisition was considered when the elasticity map was filled with colour on less than $50 \%$ of the area.
(FERRAIOLI \& al [19]; SAMIR \& al [22]).

Liver Stiffness Measurement by FibroScan- transient elastography $(\mathrm{TE})$

Measurements were performed by a single experimented evaluator, with the patient placed in dorsal decubitus, examining the right liver lobe, through intercostal spaces. Only procedures with $\geq 10$ validated measurements were considered reliable. The success rate was calculated as a percent of the validated measurements from the total number of measurements. The median value was considered representative and the results were expressed in kilopascals $(\mathrm{kPa})$. A Fibroscan was considered valid if the interquartile range was $<30 \%$ of the median value and the success rate was $>60 \%$ (BOURSIER \& al [23]).

\section{Statistical Analysis}

Variables in the present study were assessed by frequencies and descriptive statistics (categorical variables) and descriptive statistics (qualitative variables).

Patient characteristics are reported as means and standard deviations, medians (ranges), or $\mathrm{n}(\%)$ when appropriate. Continuous variables were compared with Mann-Whitney U tests. Spearman's analysis was used to investigate correlations between variables. Univariate and multivariate logistic regression analyses were performed to identify independent factors related to discordance between TE and SWE. Hazard ratios (HRs) and corresponding 95\% confidence intervals are also indicated. A two-sided $\mathrm{P}$ value of $<0.05 / 0.01$ was considered significant. All statistical analyses were performed using the SPSS 20.0 software.

\section{Results}

Between January 2017 and December 2017, 139 patients met the inclusion criteria. Sixteen patients $(11.51 \%)$ were excluded: twelve patients $(8.63 \%)$ because of unreliable liver stiffness measurement by TE (IQR > 30\% in 5 patients and no acquisitions in 7 patients) and four $(2.87 \%)$ because of unreliable liver stiffness measurement by SW (IQR > 30\%).

\section{Baseline characteristics of the study population}

A total of 123 patients were evaluated (49 men and 74 women), with a mean age of 53.56 years. Their clinical, laboratory and ultrasound descriptive parameters are summarized in Table 1.

Table 1. Baseline characteristics (biogical and ultrasound parameters) of the study population

\begin{tabular}{|c|c|c|c|c|c|}
\hline & Mean & Std dev. & Std.Err & Min & Max \\
\hline Age (years) & 53.56 & 12.532 & 1.911 & 26 & 76 \\
\hline BMI $(\mathrm{kg} / \mathrm{m} 2)$ & 31.5091 & 4.13852 & 0.62391 & 26 & 45 \\
\hline PLT & 250795.45 & 57035.109 & 8598.366 & 130000 & 348000 \\
\hline PT \% & 98.25 & 8.887 & 1.34 & 72 & 120 \\
\hline Fibrinogen & 351.52 & 72.536 & 10.935 & 165 & 486 \\
\hline $\mathrm{AST}$ & 24.2 & 10.564 & 1.593 & 12 & 71 \\
\hline ALT & 37.75 & 19.439 & 2.931 & 14 & 93 \\
\hline Alkaline phosphatase & 75.59 & 19.795 & 2.984 & 30 & 122 \\
\hline Gamma glutamyl transferase & 60.14 & 56.434 & 8.508 & 14 & 334 \\
\hline Total cholesterol $(\mathrm{mg} / \mathrm{dl})$ & 225.3 & 49.943 & 7.529 & 126 & 356 \\
\hline Triglycerides $(\mathrm{mg} / \mathrm{dl})$ & 193.2 & 119.927 & 18.08 & 42 & 485 \\
\hline $\mathrm{RL}(\mathrm{mm})$ & 140.5 & 15.687 & 2.365 & 12 & 186 \\
\hline $\mathrm{LL}(\mathrm{mm})$ & 89.5 & 14.742 & 2.222 & 53 & 121 \\
\hline $\mathrm{PV}(\mathrm{mm})$ & 12.9636 & 1.83596 & .27678 & 9.80 & 17.80 \\
\hline $\operatorname{BSD}(\mathrm{mm})$ & 120.18 & 15.015 & 2.264 & 85 & 155 \\
\hline
\end{tabular}

Abbreviations: RL: right lobe of the liver, $\mathrm{LL}=$ : left lobe of the liver, PV: portal vein diameter; BSD: bipolar spleen diameter 
Regarding non-parametrical variables, patients in the working group were divided in: grade 1 steatosis ( 9 patients $7.31 \%$ ); grade 2 steatosis (63 patients $-51.21 \%$ ) and grade 3 steatosis (51 patients $-4.46 \%)$. Liver stiffness assessment proved that TE and SWE results were correlated in 79 cases
$(64.22 \%)$. Discordance in one degree of fibrosis stage was found in 16 patients $(13.01 \%)$ and discordance in more than one degree of fibrosis stage in 28 patients $(22.76 \%)$, as shown in Figure 1.

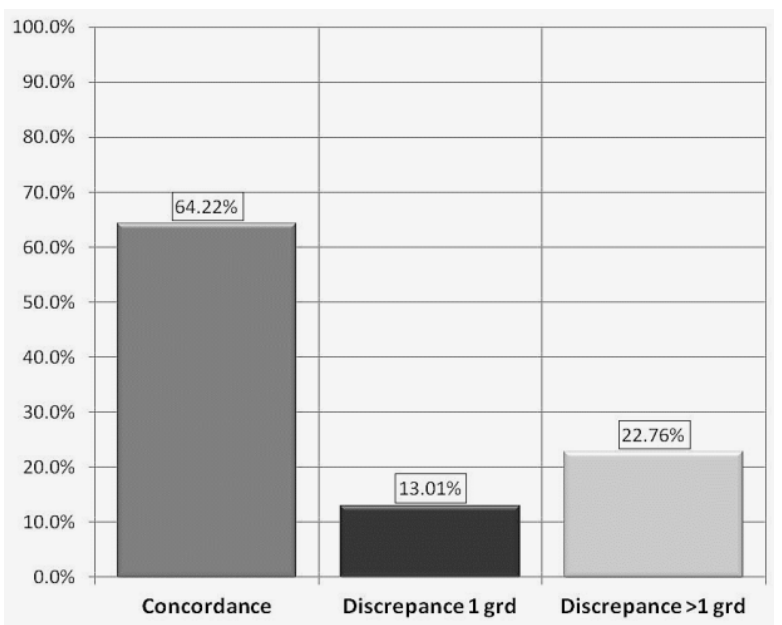

Figure 1. Overall fibrosis concordance and discordance levels in the patients included in the study.

\section{Correlations between variables}

As for the examined predictors of discordance between SWE and TE in patients with NAFLD, discordance is defined as a difference of at least 1 degree among observations performed by both methods. We analyzed demographic, clinical and biological parameters in patients with documented discrepancies for the pair of evaluation methods (TE and SWE); then we have compared those results with the parameters in concordant pairs. Statistically significant results for the up-mentioned parameters are correlated with a discordant pair of evaluation methods. We have used paired t-test and non-parametric Mann-Whitney U test that does not require assumption of normal distributions.

In the next figures, the top and bottom of the boxes represent the first and third quartiles respectively. The length of the box thus represents the interquartile range within which $50 \%$ of the values were located. The line through the middle of each box represents the median. The error bars show the minimum and maximum values (range). In our study, the SWE and TE measurements showed a concordance in patients with higher liver stiffness values (Fig 2). Pearson correlation for liver stiffness values generated by TE vs SW shows a significant association, supported by correlation coefficient $(r=0.60545)$.
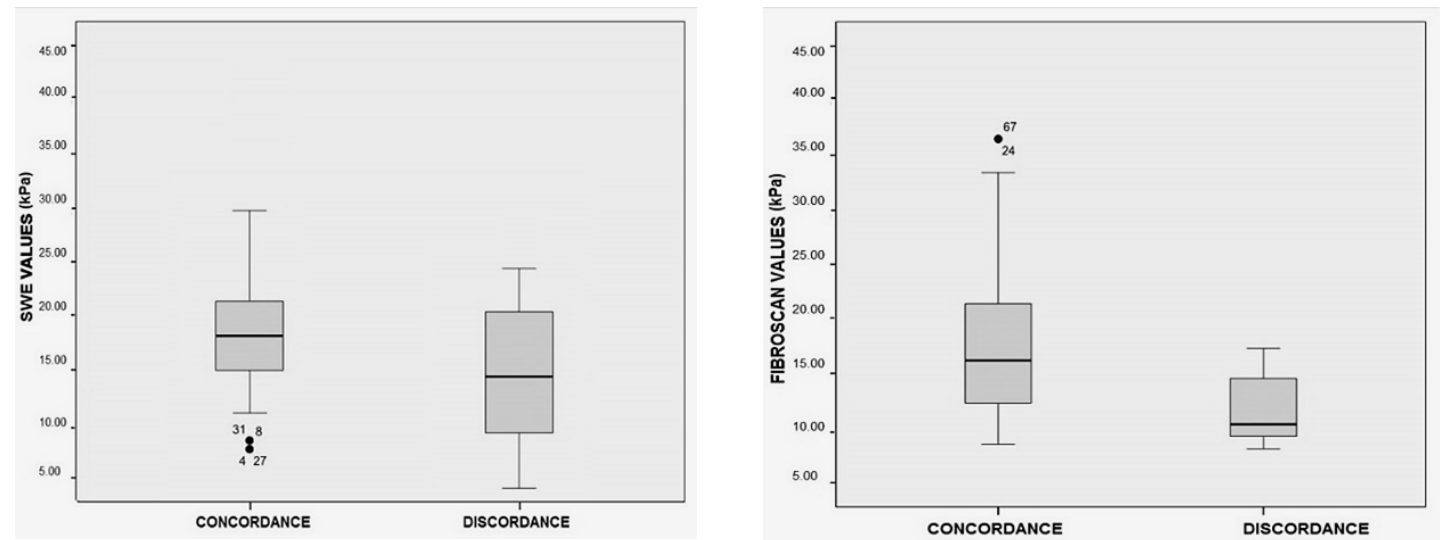

Figure 2. Concordance/discordance levels related to liver stiffness values: SWE and FibroScan measurments.

In the univariate analysis, discordance between SWE and TE in fibrosis staging (liver stiffness) was significantly influenced by: BMI, aspartate aminotransferase values and degree of liver steatosis. Results for multiple correlations regarding the predictors of discordance on the first hand and coefficients of parameters influencing differences between TE and SWE values on the other hand, are shown in Tables 2 and 3. 
Table 2. Multiple correlations in non-parametric test for predictive analysis

\begin{tabular}{|l|l|}
\hline Multiple correlations & Estimated value \\
\hline Multiple correlation coefficient $\mathrm{r}$ & 0.703538 \\
\hline Multiple $\mathrm{R}^{2}$ & 0.494965 \\
\hline $\mathrm{F}(6,1161)$ & 5.880368 \\
\hline $\mathrm{P}$ & 0.000085 \\
\hline Std.Err. of Estimate & 0.649850 \\
\hline
\end{tabular}

Table 3. Correlation coefficients of parameters influencing differences between TE and SWE values

\begin{tabular}{|c|l|l|l|l|}
\hline Partial correlation & $\begin{array}{l}\text { Correlation } \\
\text { coefficients } \\
\text { (Beta) }\end{array}$ & Std.Err. & $\mathbf{T}$ & $\begin{array}{l}\mathbf{p} \\
\mathbf{9 5 \%} \text { CI }\end{array}$ \\
\hline Intercept & & & -2.89836 & 0.005939 \\
\hline Age & -0.020559 & 0.121224 & -0.16959 & 0.866145 \\
\hline Sex & 0.133840 & 0.118238 & 1.13195 & 0.264077 \\
\hline Platelets count & -0.178353 & 0.120928 & -1.47487 & 0.147704 \\
\hline GGT & -0.088282 & 0.113211 & -0.77980 & 0.439879 \\
\hline Steatosis & 0.354682 & 0.120599 & 2.11180 & $\mathbf{0 . 0 4 0 6 9 3}$ \\
\hline BMI & 0.489139 & 0.118196 & 3.29232 & $\mathbf{0 . 0 0 2 0 2 1}$ \\
\hline ASAT & 0.502725 & 0.121724 & 3.30850 & $\mathbf{0 . 0 0 1 9 3 0}$ \\
\hline
\end{tabular}

The results of nonparametric test for predictive analisys shows that, in majority of the cases with discordance between TE and SWE values, the differences were influenced by age, BMI, level of liver enzymes (ALAT, ASAT), liver steatosis and platelets count. The analysis of the partial correlation coefficients indicated that a significant influence in discordance of fibrosis stage was exercised by the ASAT $\left(r_{\text {partial }}=0.50, \mathrm{p}<0.01\right)$, followed by the value of BMI $\left(\mathrm{r}_{\text {partial }}=0.48, \mathrm{p}<0.01\right)$ and the last predictive factor steatosis degree $\left(r_{\text {partial }}=0.35, \mathrm{p}<0.05\right)($ Table 3$)$.

\section{Discussions}

In our series SWE applicability was $(97.13 \%)$ significantly higher than that TE (91.36\%). SWE applicability was greater than TE applicability in obese patients and the rate of failure appears to be lower than for TE. These same results were reported by other authors (POYNARD \& al [20], ELKRIEF \& al [24]; WONG \& al [25]; BOTA \& al [26]).

The prevalence of significant discordance (more than 1 degree) in our study was higher $(22.76 \%)$ than that in other studies (THIELE \& al [27], GERBER \& al [28]; KIM \& al [29]).

It is possible that these results are due to the fact that SW elastography offers the possibility to choose the placement of ROI within the color stiffness box and also to include or exclude each single measurement when determining the final value. Furthermore, it is a greater potential for the operator to influence the final results than when using Fibroscan, where this variability is not applicable (LI \& al [30], CASSINOTTO \& al [31]; MULLER \& al [32]).

Both methods are comparable in most cases for staging advanced liver fibrosis (F3) and diagnosing cirrhosis (F4). In our study, SWE values are well correlate with transient elastography values in cases of advanced fibrosis (F3-F4) but not as well at low levels of liver fibrosis (F0-F2) Related to this aspect, most previous reports noted the high degree of concordance for advanced fibrosis stages (F3-F4) (KIM \& al [29], LI \& al [30]; PISCAGLIA $\&$ al [33]).
High values of liver stiffness $(>15 \mathrm{kPa}$ ) showed independent positive association with concordance between TE and SWE, fact that has been reported by other authors as well (GERBER \& al [28]; JEONG \& al [34]). Similarly to other studies, in the study we performed, high body mass index (BMI) was an important factor in predicting the discordance between TE and SWE (PISCAGLIA \& al [33]; JEONG \& al [34], YOON \& al [35]; CHANG \& al [36], PETTA \& al [37]).

The second factor that may influence the concordance between the two methods is necroinflammatory activity, which is closely related to ALT/AST level. Our results show that the discordance in fibrosis staging was influenced by the ASAT values. Results from other studies have identified elevated liver enzymes as a predictor of discordance. No effect of the high level of liver enzymes was seen on liver stiffness measurements in other previously reported studies (ELKRIEF \& al [24], PISCAGLIA \& al [33]).

Impact of steatosis on discordance between TE and SWE was also evaluated in the present study, concludingsimilarly to other studies - that the presence of steatosis influences conducting liver stiffness measurement, in order to assess fibrosis (GAIA \& al [38], PETTA \& al [39]). On the contrary, in the study performed by Deffieux et al elasticity measurements were not influenced by the degree of steatosis (DEFFIEUX \& al [40]). In our study lot, liver stiffness degree did not correlate with platelet counts in either the univariate or multivariate analysis. This result is similar to those observed in other studies using shear wave or transient elastography techniques (OLIVARESGAZCA \& al [41]).

For liver stiffness measurements by TE, there was a possible overestimation, related to the necroinflammatory activity, while for estimated fibrosis using SWE, there was a possible underestimation, due to the high BMI and presence of liver steatosis. Our study has limitations: the study population had a high prevalence of obesity, which could be a technical limitation and the analysis was performed in a relatively small number of patients; thus, these results need to be validated in larger studies. 


\section{Conclusions}

SWE and transient elastography appear as comparably reliable methods for non-invasive assessment of liver fibrosis. However, there are possible discrepancies in evaluating fibrosis stage using TE and SWE, such differences being significantly influenced by BMI, level of necroinflammatory activity and liver steatosis. Both methods show better reliability for the detection of advanced fibrosis and cirrhosis (F3-F4) when compared to the detection of incipient or significant fibrosis (F0-F2).

Although alternative SWE seem to overcome limitations of TE, their quality criteria for high sensitivity interpretation have not yet been well defined.

Given the socio-economic impact determined by the increased morbidity and mortality caused by chronic hepatic diseases, the development of non-invasive, accessible and precise methods is essential. These methods would prove helpful in establishing an early diagnostic, which in turn would lead to an efficient treatment.

In the future, formulas that combine methods of elastometry and biomarkers that identify hepatic fibrosis will completely replace hepatic biopsy in establishing the severity of hepatic illness, both in NAFLD and in other chronic hepatic diseases.

\section{References}

1. MARCHESINI G, BUGIANESI E, FORLANI G, et al. Nonalcoholic fatty liver, steatohepatitis, and the metabolic syndrome, Hepatology 2003; 37, pp. 917-923.

2. ANSTEE QM, MCPHERSON S, DAY CP. How big a problem is non-alcoholic fatty liver disease?, BMJ 2011; 343, d3897.

3. MCPHERSON S, HARDY T, HENDERSON E, et al. Evidence of NAFLD progression from steatosis to fibrosing-steatohepatitis using paired biopsies: Implications for prognosis and clinical management, J Hepatol 2015; 62, pp. 1148-1155.

4. BELLENTANI S, SCAGLIONI F, MARINO M, et al. Epidemiology of non-alcoholic fatty liver disease, Dig Dis 2010; 28; pp. 155-161.

5. MACHADO MV, CORTEZ-PINTO H. Non-invasive diagnosis of non-alcoholic fatty liver disease. A critical appraisal. Journal of Hep 2013; 58; pp. 1007-1019.

6. ARMSTRONG MJ, HOULIHAN DD, BENTHAM L, et al. Presence and severity of non-alcoholic fatty liver disease in a large prospective primary care cohort, J Hepatol 2012; 56, pp. 234-240.

7. EKSTEDT M, HAGSTROM H, NASR P, FREDRIKSON M, et al. Fibrosis stage is the strongest predictor for disease-specific mortality in NAFLD after up to 33 years of follow-up. Hepatology 2015; 61, 1547-1554. doi: 10.1002/ hep. 27368

8. ANGULO P, KLEINER DE, DAM-LARSEN S, ADAMS M, et al. Liver fibrosis, but no other histologic features, is associated with long-term outcomes of patients with nonalcoholic fatty liver disease. Gastroenterology 2015; 149, 389-97.e10. doi: 10.1053/j.gastro.2015.04.043

9. NIH Consensus Statement on Management of Hepatitis C. Hepatology 2002; 36, pp. S3-S20.

10. CASTERA L. Noninvasive methods to assess liver disease in patients with hepatitis B or C. Gastroenterology 2012, 142, pp. 1293-1302.

11. RATZIU V, MASSARD J, CHARLOTTE F, et al. Diagnostic value of biochemical markers (FibroTestFibroSURE) for the prediction of liver fibrosis in patients with non-alcoholic fatty liver disease. $B M C$ Gastroenterology 2006; 6:6, 1471-230X/6/6.

12. EASL-ALEH Clinical Practice Guidelines: Noninvasive tests for evaluation of liver disease severity and prognosis. Journal of Hepatology 2015; vol. 63, pp. 237-264.

13. SANDRIN L. Transient elastography: a new noninvasive method for assessment of hepatic fibrosis. Ultrasound Med Biol 2003; 29, pp. 1705-1713.

14. FRIEDRICH-RUST M. Performance of transient elastography for the staging of liver fibrosis: a metaanalysis. Gastroenterology 2008; 134(4), pp. 960-974.

15. MYERS RP. Prevalence, risk factors and causes of discordance in fibrosis staging by transient elastography and liver biopsy. Liver Int 2010; 30, pp. 1471-1480.

16. TAMANO M, KOJIMA K, AKIMA T, et al. The usefulness of measuring liver stiffness by transient elastography for assessing hepatic fibrosis in patients with various chronic liver diseases. Hepatogastroenterology. 2012 May; 59(115): pp. 826-30. doi: 10.5754/hge11255

17. CASTERA L, FORNS X, ALBERTI A. Noninvasive evaluation of liver fibrosis using transient elastography. J Hepatol. 2008; 48: pp. 835-847.

18. SIGRIST RMS, LIAU J, KAFFAS AE, et al. Ultrasound Elastography: Review of Techniques and Clinical Applications. Theranostics. 2017; 7(5): pp. 1303-1329. doi:10.7150/thno. 18650

19. FERRAIOLI G, TINELLI C, DAL BELLO B, et al. Accuracy of real-time shear wave elastography for assessing liver fibrosis in chronic hepatitis C: a pilot study. Hepatology. 2012; 56(6): pp. 2125-33.

20. POYNARD T, MUNTEANU M, LUCKINA E, et al. Liver fibrosis evaluation using real-time shear wave elastography: applicability and diagnostic performance using methods without a gold standard. J Hepatol 2013;58: pp. 928-935.

21. CHALASANI N, YOUNOSSI Z, LAVINE JE, et al. The diagnosis and management of non-alcoholic fatty liver disease:practice Guideline by the American Association for the study of Liver Disease, American College of Gastroenterology, and the American Gastroenterological Association. Hepatology 2012; 55; pp. 2005-2023. 
22. SAMIR AE, DHYANI M, VIJ A, et al. Shear-Wave Elastography for the Estimation of Liver Fibrosis in Chronic Liver Disease: Determining Accuracy and Ideal Site for Measurement. Radiology. 2014:140839

23. BOURSIER J, ZARSKI JP, DE LEDINGHEN S et al. (Multicentric Group from ANRS/HC/EP23 FIBROSTAR Studies). Determination of reliability criteria for liver stiffness evaluation by transient elastography. Hepatology. 2013 Mar; 57(3): pp. 1182-91.

24. ELKRIEF L, RAUTOU P, RONOT M, et al. Prospective comparison of spleen and liver stiffness by using shear-wave and transient elastography for detection of portal hypertension in cirrhosis. Radiology 2015; 275, pp. 589-598. doi: 10.1148/radiol.14141210

25. WONG GL, WONG VW, CHIM AM, et al. Factors associated with unreliable liver stiffness measurement and its failure with transient elastography in the Chinese population. J. Gastroenterol. Hepatol. 2011; 26, pp. 300-305. doi: 10.1111/j.1440-1746. 2010.06510.x

26. BOTA S, PATERNOSTRO R, ETSCHMAIER A. Performance of 2-D shear wave elastography in liver fibrosis assessment compared with serologic tests and transient elastography in clinical routine. Ultrasound Med. Biol. 2015; 41, 2340-2349. doi: 10.1016/j.ultrasmedbio.2015.04.013

27. THIELE M, DETLEFSEN S, SEVELSTED MØLLER L, et al. Transient and 2-dimensional shear-wave elastography provide comparable assessment of alcoholic liver fibrosis and cirrhosis. Gastroenterology 2016; 150, pp. 123-133. doi: 10.1053/j.gastro.2015.09.040

28. GERBER L, KASPER D, FITTING D, et al. Assessment of liver fibrosis with 2-D shear wave elastography in comparison to transient elastography and acoustic radiation force impulse imaging in patients with chronic liver disease. Ultrasound Med Biol. 2015 Sep; 41(9): pp. 2350-9. doi: 10.1016/j. ultrasmedbio.2015.04.014. Epub 2015 Jun 24.

29. KIM H J, LEE H K, CHO J H, et al. Quantitative comparison of transient elastography (TE), shear wave elastography (SWE) and liver biopsy results of patients with chronic liver disease. Journal of Physical Therapy Science. 2015; 27(8): pp. 24652468. doi:10.1589/jpts.27.2465

30. LI C, ZHANG C, LI J, HUO H, SONG D. Diagnostic Accuracy of Real-Time Shear Wave Elastography for Staging of Liver Fibrosis: A MetaAnalysis. Med Sci Monit. 2016 Apr 22; 22:1349-59. Epub 2016 Apr 22.

31. CASSINOTTO C, LAPUYADE B, MOURIES A, et al. Non-invasive assessment of liver fibrosis with impulse elastography: comparison of Supersonic
Shear Imaging with ARFI and FibroScan R. J. Hepatol. 2014; 61, pp. 550-557. doi: 10.1016/ j.jhep.2014.04.044

32. MULLER M, GENNISSON JL, DEFFIEUX T, et al. Quantitative viscoelasticity mapping of human liver using supersonic shear imaging: preliminary in vivo feasibility study. Ultrasound Med Biol. 2009; 35: pp. 219-229.

33. PISCAGLIA F, SALVATORE V, MULAZZANI L. et al. Ultrasound Shear Wave Elastography for Liver Disease. A Critical Appraisal of the Many Actors on the Stage. Ultraschall Med. 2016 Feb; 37(1): pp. 1-5. Epub 2016 Feb 12.

34. JEONG JY, KIM TY, SOHN J H, et al. Real time shear wave elastography in chronic liver diseases: accuracy for predicting liver fibrosis, in comparison with serum markers. World J. Gastroenterol. 2014; 20, 13920-13929. doi: 10.3748/wjg.v20.i38.13920

35. YOON JH, LEE JM, HAN JK, et al. Shear wave elastography for liver stiffness measurement in clinical sonographic examinations: evaluation of intraobserver reproducibility, technical failure, and unreliable stiffness measurements. J Ultrasound Med. 2014 Mar; 33(3): pp. 437-47.

36. CHANG PE, GOH GB B, NGU JH, et al. Clinical applications, limitations and future role of transient elastography in the management of liver disease. World J Gastrointest Pharmacol Ther. 2016;7(1): pp. 91-106. doi:10.4292/wjgpt.v7.i1.91

37. PETTA S, DI MARCO V, CAMMÀ C, et al. Reliability of liver stiffness measurement in nonalcoholic fatty liver disease: the effects of body mass index. Aliment. Pharmacol. Ther. 2011; 33, pp. 13501360. doi: 10.1111/j.1365-2036.2011.04668

38. GAIA S, CARENZI S, BARILLI AL, et al. Reliability of transient elastography for the detection of fibrosis in non-alcoholic fatty liver disease and chronic viral hepatitis. J Hepatol. 2011; 54: pp. 64-71.

39. PETTA S. The severity of steatosis influences liver stiffness measurement in patients with nonalcoholic fatty liver disease. Hepatology 2015. 62(4), pp. 1101-1110.

40. DEFFIEUX T, GENNISSON JL, BOUSQUET L, et al. Investigating liver stiffness and viscosity for fibrosis, steatosis and activity staging using shear wave elastography. J. Hepatol. 2015; 62, 317-324. doi: 10.1016/j.jhep.2014.09.020

41. OLIVARES-GAZCA JC, NUÑEZ-CORTES AK, MENDEZ-HUERTA MA, et al. More on the thrombocytopenia of the non-alcoholic fatty liver disease. Hematology 2017; vol. 22, Iss. 5, pp. 316-319. 